\title{
STATISTICAL DATA ANALYSIS METHOD FOR MULTIZONAL AIRFLOW MEASUREMENT USING MULTIPLE KINDS OF PERFLUOROCARBON TRACER GAS
}

\author{
Hiroyasu OKUYAMA ${ }^{1 \dagger}$ \\ Shin-ichi TANABE ${ }^{2}$ \\ Yoshinori ONISHI \\ Seiichi KASHIHARA ${ }^{3}$ \\ ${ }^{1}$ Institute of Technology, Shimizu corp., Tokyo, Japan \\ ${ }^{2}$ Dept. of Architecture, Waseda University, Tokyo, Japan \\ ${ }^{3}$ Fundamental Technology Dept.,Asahi Kasei Homes Corporation, Tokyo, Japan
}

\begin{abstract}
Conventional multiple types of perfluorocarbon tracers use the number of gases equal to the number of zones $(n)$. The possible $n \times n+n$ airflows are solved from the mass balance of the gas and the airflow balance. However, some airflows may not occur because of the actual interzonal geometry, and the introduction of unnecessary, unknown parameters can impair the accuracy of the estimations. Also, various error factors often yield an irrational negative airflow rate. Conventional methods have insufficient procedure for the evaluation of error. This study describes a way to use the least-squares technique to improve the precision of estimates and to evaluate reliability. From the equations' residual the error variance and covariance matrix $\boldsymbol{\Lambda}_{q}$ of the estimated airflow rate error is deduced and also the coefficient of determinant using the residual square sum and total variation is introduced. Further the error matrix ${ }_{m} \boldsymbol{\Lambda}_{q}$ caused by the measurement error of the gas concentration and gas emission rate is deduced. The discrepancy ratio of the model premise is defined by dividing the former diagonal elements by the latter ones. Moreover the index of irrationality of the estimated negative airflow rate is defined based on the difference of the three estimation methods results.
\end{abstract}

\section{KEYWORDS}

Multizonal Airflow Measurement, Perfluorocarbon Tracer Gas, Least Squares, Error Analysis

\section{INTRODUCTION}

A multizonal airflow measurement method uses single type of gas (Okuyama 1990,1992). This method requires a certain device to be set in the building to actively cause the gas concentration to be changed with time, and measure and record the results. On the other hand, a simple measurement method called PFT (Perfluorocarbon Tracer) uses simple tools. Initially (Stymne and Boman 1994) this method was used for single-zone applications but recently some instances using 3 types (Higashita and Tanabe et al. 2004) or 4 types (Mihara and Yoshino et al. 2006) of PFTs for measuring multizonal airflow have been reported. Generally in case of $n$ zones, there may be $n(n+1)$ airflows to be estimated. Long-term average gas mass balance equations are described for each zone. When $n$ types of different PFTs are diffused in each zone, $n^{2}$ balance equations are obtained and $n$ airflow balance equations are also established. Such a conventional conception that equations of total $n(n+1)$ equivalent to the maximum unknown quantities can be obtained and solved is called a deterministic method.

Conventional method, however, presents no reliability evaluation method that is properly executed so that the premises for the measurement are met. The premises mean that the concentration is uniform, the airflow is constant in each zone, and other conditions. Conventional error evaluations only took measurement errors into consideration and errors due to unsatisfied premises were not taken into

†Corresponding Author: Tel: $81+3+3820+6438$, Fax: $81+3+3820+5955$

E-mail address: okuyama@shimz.co.jp 
account despite of their significance. In addition even airflow not existent should be obtained as an unknown quantity for the reason of geometrical relation between zones. Therefore, irrational negative airflows are often obtained with conventional methods.

In order to resolve those problems, we should regard them as problems of optimization after acknowledging the existence of errors of equations, to obtain such an airflow as to minimize those errors in the positive range and obtain the optimum solution by a statistical method utilizing the more equations than the number of unknown quantities from measurements as in the least squares method. In this paper, newly contrived error evaluation indices are described with the same models and solutions as reported in the previous paper (Okuyama 1990).

\section{BASIC MATHEMATICAL MODEL}

When there are $n$ zones indoors, a balance equation of some type of tracer gas in the $i$-th zone is described as follows. The open air is considered to be a type of zone and its zone No. is defined as $n+1$. Airflow from the zone $j$ to zone $i$ is defined and noted as $q_{i j}$. Gas concentration in the zone $i$ is defined and noted as $c_{i}$, rate of gas generated as $g_{i}$, and effective mixture volume as $v_{i}$.

$$
v_{i} \cdot \dot{c}_{i}=\sum_{j=1}^{n+1} q_{i, j} \cdot c_{j}-\sum_{j=1}^{n+1} q_{j, i} \cdot c_{i}+g_{i}
$$

By integrating this by the term $T$, following equation can be described. It should be assumed here that both $q$ and $v$ are constant to the extent that they can be considered to be term average values with a bar.

$$
\bar{v}_{i} \cdot \frac{1}{T}\left[c_{i}(T)-c_{i}(0)\right]=\sum_{j=1}^{n+1} \bar{q}_{i, j} \cdot \frac{1}{T} \int_{0}^{T} c_{j} d t-\sum_{j=1}^{n+1} \bar{q}_{j, i} \cdot \frac{1}{T} \int_{0}^{T} c_{i} d t+\frac{1}{T} \int_{0}^{T} g_{i} d t
$$

By securing enough term $T$, the left side can be approximated to 0 . Result of time integral calculus of concentration on the right side divided by $T$ is average term concentration. Rate of gas diffused also becomes average term diffusion rate. These are expressed with a bar in the same way. As a result, following approximation equation can be obtained.

$$
\sum_{j=1}^{n+1} \bar{q}_{i, j} \cdot \bar{c}_{j}-\sum_{j=1}^{n+1} \bar{q}_{j, i} \cdot \bar{c}_{i}+\bar{g}_{i}=0
$$

As this theory is based on a statistical method, measured values should be obtained under as many conditions as possible. Number of gas types, $k$, should be more than the number of zones, $n k$. Measurement term, $p$, should be more than 1, np. Number of point, 1, in the zone to measure the concentration should be more than $1, n l$. In this way, the estimation accuracy can be improved and errors can be properly evaluated. Equation (3) can be rewritten as follows using the condition Nos. $p$, $k$, and $l$.

$$
\sum_{j=1}^{n+1} \bar{q}_{i, j}{ }_{p, k, l} \bar{c}_{j}-\sum_{j=1}^{n+1} \bar{q}_{j, i} \cdot{ }_{p, k, l} \bar{c}_{i}+{ }_{p, k} \bar{g}_{i}=0
$$

This can be expressed as a matrix with the equation (7) by defining the concentration and gas generation rate vectors respectively with the equation (5) and (6). Here, $t$ on the left shoulder means transpose of matrix.

$$
\begin{array}{rl}
p, k, l & \mathbf{c}={ }^{t}{ }_{p, k, l} \bar{c}_{1}, \cdots,{ }_{p, k, l} \bar{c}_{n}, p, k, l \\
\left.\bar{c}_{n+1}\right) & \operatorname{size}(n+1) \\
p, k & \mathbf{g}={ }^{t}\left({ }_{p, k} \bar{g}_{1}, \cdots,{ }_{p, k} \bar{g}_{n}\right) \operatorname{size}(n) \\
& \mathbf{Q}_{p, k, l} \mathbf{c}+{ }_{p, k} \mathbf{g}=\mathbf{0}
\end{array}
$$


Concentration in the open air is represented to be non 0 generally. Matrix $\mathbf{Q}$ is $n x(n+1)$ including the airflow. In the rightmost column, airflow entering from the open air into each zone is positioned.

\section{REGRESSION EQUATION AND SOLUTION OF LEAST SQUARES METHOD}

In order to obtain solutions for multiple airflows, vector $\mathbf{q}$ including them is defined as shown in the equation (8). Elements in the vector can be freely ordered. Equation (7) is changed to an equation for the airflow q. Elements of the matrix $\mathbf{Z}$ of $n x n q$ for the vector $\mathbf{q}$ of size $n q$ are determined as follows. Here $n q$ is the number of airflows of unknown quantities. When the $m$-th element in the vector $\mathbf{q}$ is $q_{i j}$, $i$-th row $m$-th column element or $(i, m)$ element in the matrix is $c_{j}$ and $(j, m)$ element is $-c_{j}$. If $i$ or $j$ exceeds $n$, they are excluded.

$$
\mathbf{q}={ }^{t}\left(\cdots, \bar{q}_{i, j}, \cdots\right)(8) \quad{ }_{p, k, l} \mathbf{Z} \cdot \mathbf{q}+{ }_{p, k} \mathbf{g}=\mathbf{0}(9)
$$

Conditional expression of airflow balance should be also considered. For instance, the airflow balance equation in the $i$-th zone is expressed with the equation (10). This is described for all zones and rewritten as follows with the vector $\mathbf{q}$ of the airflow. Elements of the matrix $\mathbf{C}$ of $n \times n q$ for $\mathbf{q}$ are determined as follows. When the $m$-th element in $\mathbf{q}$ is $q_{i j},(i, m)$ element in this matrix is 1 and $(j, m)$ element is -1 . They are excluded if $i$ or $j$ exceeds $n$.

$$
\sum_{j=1}^{n+1} \bar{q}_{i, j}-\sum_{j=1}^{n+1} \bar{q}_{j, i}=0 \quad(10) \quad \mathbf{C} \cdot \mathbf{q}=\mathbf{0}
$$

Error $\mathbf{e}$ of the equation of the least squares method is defined with the following equation with $\mathbf{Z}, \mathbf{g}$ and $\mathbf{C}$ as described above. Matrix $\mathbf{F}$ and vector $\mathbf{d}$ are defined as shown in the following equation to simplify the description.

$$
p, k, l=\left[\begin{array}{c}
p, k, l \\
\mathbf{Z} \\
\mathbf{C}
\end{array}\right] \cdot \hat{\mathbf{q}}+\left[\begin{array}{c}
p, k \\
\mathbf{0}
\end{array}\right]={ }_{p, k, l} \mathbf{F} \cdot \hat{\mathbf{q}}+{ }_{p, k} \mathbf{d}
$$

Evaluation function $J$ of this error $\mathbf{e}$ is defined with the following equation.

$$
J=\sum_{p=1}^{n p} \sum_{k=1}^{n k} \sum_{l=1}^{n l}{ }_{p, k, l}^{t} \mathbf{e}{ }_{p, k, l} \mathbf{e}=\sum_{p=1}^{n p} \sum_{k=1}^{n k} \sum_{l=1}^{n l}{ }^{t}\left({ }_{p, k, l} \mathbf{F} \cdot \mathbf{q}+{ }_{p, k} \mathbf{d}\right) \cdot\left({ }_{p, k, l} \mathbf{F} \cdot \mathbf{q}+{ }_{p, k} \mathbf{d}\right)
$$

The solution of the least squares method is derived from the following equation by differentiating $J$ by $\mathbf{q}$ as 0 .

$$
\frac{\partial J}{\partial \mathbf{q}}=2 \sum_{p=1}^{n p} \sum_{k=1}^{n k} \sum_{l=1}^{n l}{ }_{p, k, l}{ }^{t} \mathbf{F} \cdot{ }_{p, k, l} \mathbf{F} \cdot \mathbf{q}+2 \sum_{p=1}^{n p} \sum_{k=1}^{n k} \sum_{l=1}^{n l}{ }_{p, k, l}{ }^{t} \mathbf{F} \cdot{ }_{p, k} \mathbf{d}=\mathbf{0}
$$

From this equation (14), solution of the least squares method is calculated with the following equation.

$$
\hat{\mathbf{q}}=-\left(\sum_{p=1}^{n p} \sum_{k=1}^{n k} \sum_{l=1}^{n l}{ }_{p, k, l^{t}}{ }^{\mathbf{F}} \cdot{ }_{p, k, l} \mathbf{F}\right)^{-1} \cdot\left(\sum_{p=1}^{n p} \sum_{k=1}^{n k} \sum_{l=1}^{n l}{ }_{p, k, l}{ }^{t} \mathbf{F} \cdot{ }_{p, k} \mathbf{d}\right)
$$

This is a solution of a normal least squares method. In an actual situation where various errors may arise, the airflow is often estimated to be negative. An optimum q with the minimum $J$ in the non-negative constraints can be obtained with the algorism of the non-negative least squares method (Lawson and Hanson 1974). The basic equation is the same also in this case.

\section{EQUATION RESIDUAL ANALYSIS AND ESTIMATED ERROR MATRIX}

One of the keys to evaluate the estimated errors is the residual of equation. Not only measurement errors of concentration or diffusion quantities, various causes of errors such as structural differences of 
equation models and actual phenomena are finally represented in the residual of equation. Residual $v$ is defined with the following equation.

$$
{ }_{p, k, l} \mathbf{v}={ }_{p, k, l} \mathbf{F} \cdot \hat{\mathbf{q}}+{ }_{p, k} \mathbf{d}
$$

Matrix of expected value of equation residual is defined with the following equation. Here, $n t$ is total number of various conditions by $p, k$, and $l$.

$$
E\left(\mathbf{e}^{t} \mathbf{e}\right) \cong \frac{1}{n t} \sum_{p=1}^{n p} \sum_{k=1}^{n k} \sum_{l=1}^{n l}{ }_{p, k, l} \mathbf{v} \cdot{ }_{p, k, l}^{t} \mathbf{v}
$$

Estimated error variance - covariance matrix of airflow can be described with the following equation as an error propagation from this matrix of expected value of error.

$$
\begin{gathered}
\boldsymbol{\Lambda}_{\mathbf{q}}=\left(\sum_{p=1}^{n p} \sum_{k=1}^{n k} \sum_{l=1}^{n l}{ }_{p, k, l}^{t} \mathbf{F}{ }_{p, k, l} \mathbf{F}\right)^{-1} \cdot\left(\sum_{p=1}^{n p} \sum_{k=1}^{n k} \sum_{l=1}^{n l}{ }_{p, k, l}^{t} \mathbf{F} \cdot E\left(\mathbf{e} \cdot{ }^{t} \mathbf{e}\right)_{p, k, l} \mathbf{F}\right)^{t} \\
\cdot\left\{\left(\sum_{p=1}^{n p} \sum_{k=1}^{n k} \sum_{l=1}^{n l}{ }_{p, k, l}^{t} \mathbf{F}{ }_{p, k, l} \mathbf{F}\right)^{-1}\right\}
\end{gathered}
$$

The diagonal element is error variance of the airflow and the non-diagonal element is covariance. Calculation equation of the coefficient of determinant is shown below. Residual sum square, $s(q)$, of all the conditions $p, k$, and $l$ is defined with the equation (19). Since the gas diffusion rate is considered to vary depending on the term $p$ and gas type $k$ but considered to have no relation with the gas absorption position $l, \mathbf{y}$ of the equation (20) is defined.

$$
s(\hat{\mathbf{q}})=\sum_{p=1}^{n p} \sum_{k=1}^{n k} \sum_{l=1}^{n l}{ }_{p, k, l}^{t} \mathbf{v} \cdot{ }_{p, k, l} \mathbf{v} \quad(19), \quad{ }_{p, k, l} \mathbf{y}={ }_{p, k} \mathbf{d}
$$

Taking the difference from the term average of the gas diffusion rate vector $\mathbf{y}$, the value taking the sum of squares on various conditions is expressed with the equation (21) called the total variation $s_{y}$. Coefficient of determinant, COD, is calculated with the equation (22)

$$
\begin{aligned}
s_{y}=\sum_{p=1}^{n p} \sum_{k=1}^{n k} \sum_{l=1}^{n l}{ }^{t}\left({ }_{p, k, l} \mathbf{y}-\overline{\mathbf{y}}\right) \cdot\left({ }_{p, k, l} \mathbf{y}-\overline{\mathbf{y}}\right)= & \sum_{p=1}^{n p} \sum_{k=1}^{n k} \sum_{l=1}^{n l}{ }_{p, k, l}{ }_{l}^{t} \mathbf{y}_{p, k, l} \mathbf{y} \\
& -\frac{1}{n t}\left(\sum_{p=1}^{n p} \sum_{k=1}^{n k} \sum_{l=1}^{n l}{ }_{p, k, l} t \mathbf{y}\right) \cdot\left(\sum_{p=1}^{n p} \sum_{k=1}^{n k} \sum_{l=1}^{n l}{ }_{p, k, l} \mathbf{y}\right)
\end{aligned}
$$

$$
C O D=1-\frac{s(\hat{\mathbf{q}})}{s_{y}}
$$

\section{INDEX OF DISCREPANCY FROM MODEL PREMISES}

Error propagation to estimated parameters is described only from the measurement errors of gas diffusion rate and gas concentration. These errors are underestimated than actual values but appropriateness of model premises can be evaluated by comparing with the errors estimated from the equation residual in the previous section. An equation is defined using only measurement errors corresponding to the equation (16) of the equation residual. Measured values are considered to be true values added with the error $\mathbf{s}$. Considering that true values make the equation errors 0 , following equation can be expressed. Lower half of the vector represents constraints on the airflow balance and 
can be considered to be $\mathbf{0}$ as it has no relation with measurement errors.

$$
{ }_{p, k} \boldsymbol{\varepsilon}=\left[\begin{array}{c}
\mathbf{Q} \cdot{ }_{p, k} \mathbf{s}_{c}+{ }_{p, k} \mathbf{s}_{g} \\
\mathbf{C} \cdot \hat{\mathbf{q}}
\end{array}\right]=\left[\begin{array}{c}
\mathbf{Q}_{p, k} \mathbf{s}_{c}+{ }_{p, k} \mathbf{s}_{g} \\
\mathbf{0}
\end{array}\right]
$$

Expected value matrix of equation errors due to measurement errors can be calculated with the error standard deviation $\sigma_{\mathrm{c}}$ and $\sigma_{\mathrm{g}}$ as the characteristics of the instruments with the equation (24). In this case, such features were utilized that the covariance between the errors ${ }_{p, k} s_{c}$ and ${ }_{p, k} s_{g}$ is 0 and the covariance between elements in these two vectors is also 0. "diag" indicates a matrix composed of only the diagonal elements in the matrix in this ( ). Accordingly, the expected value matrix of ${ }_{p, k} \varepsilon$ added with the vector of the lower half can be calculated with the equation (25). Estimated error matrix of the airflow is calculated with the equation (26).

$$
\begin{aligned}
& { }_{p, k} E\left({ }_{p, k} \boldsymbol{\varepsilon}_{u} \cdot{ }_{p, k} \boldsymbol{\varepsilon}_{u}\right)=\mathbf{Q}_{p, k} E\left({ }_{p, k} \mathbf{s}_{c} \cdot{ }_{p, k}^{t} \mathbf{s}_{c}\right) \cdot{ }^{t} \mathbf{Q}+_{p, k} E\left({ }_{p, k} \mathbf{s}_{g} \cdot{ }_{p, k}^{t} \mathbf{s}_{g}\right) \\
& =\mathbf{Q} \cdot_{p, k} E\left(\operatorname{diag}\left({ }_{p, k} \boldsymbol{\sigma}_{c} \cdot{ }_{p, k}^{t} \boldsymbol{\sigma}_{c}\right)\right)^{t} \mathbf{Q}+{ }_{p, k} E\left(\operatorname{diag}\left({ }_{p, k} \boldsymbol{\sigma}_{g} \cdot{ }_{p, k}^{t} \boldsymbol{\sigma}_{g}\right)\right) \\
& { }_{p, k} E\left({ }_{p, k} \boldsymbol{\varepsilon}_{p, k} \boldsymbol{\varepsilon}\right)=\left[\begin{array}{cc}
{ }_{p, k} E\left({ }_{p, k} \boldsymbol{\varepsilon}_{u} \cdot{ }_{p, k} \boldsymbol{\varepsilon}_{u}\right) & \mathbf{0} \\
\mathbf{0} & \mathbf{0}
\end{array}\right] \\
& { }_{m} \boldsymbol{\Lambda}_{\mathbf{q}}=\left(\sum_{p=1}^{n p} \sum_{k=1}^{n k} \sum_{l=1}^{n l}{ }_{p, k, l}^{t} \mathbf{F} \cdot_{p, k, l} \mathbf{F}\right)^{-1} \cdot\left(\sum_{p=1}^{n p} \sum_{k=1}^{n k} \sum_{l=1}^{n l}{ }_{p, k, l}^{t} \mathbf{F}{ }_{p, k} E\left({ }_{p, k} \boldsymbol{\varepsilon}_{p, k}{ }_{p, k}^{t} \boldsymbol{\varepsilon}\right)_{p, k, l} \mathbf{F}\right) . \\
& \left\{\left(\sum_{p=1}^{n p} \sum_{k=1}^{n k} \sum_{l=1}^{n l}{ }_{p, k, l}^{t} \mathbf{F}{ }_{p, k, l} \mathbf{F}\right)^{-1}\right\}
\end{aligned}
$$

It can be judged by comparing the diagonal element size of ${ }_{m} \boldsymbol{\Lambda}_{q}$ with that of $\boldsymbol{\Lambda}_{q}$ to what degree of the premises of the mathematical model is satisfied with actual phenomena. Here, the $j$-th diagonal element of ${ }_{m} \boldsymbol{\Lambda}_{q}$ is expressed with ${ }_{m} \sigma_{\lambda j j}^{2}$, and the $j$-th diagonal element of $\boldsymbol{\Lambda}_{q}$ is with $\sigma_{\lambda j j}^{2}$. Taking the square root of these diagonal elements, discrepancy rate $\beta$ of the model premises of the following equation and its average value are defined.

$$
\beta_{j}=\frac{\sigma_{\lambda_{j, j}}}{{ }_{m} \sigma_{\lambda, j}} \text { (27) } \quad \bar{\beta}=\frac{1}{n q} \sum_{j=1}^{n q} \beta_{j}
$$

\section{IRRATIONALITY INDEX OF ESTIMATED NEGATIVE AIRFLOW}

Results of the non-negative least squares method and the normal least squares method accord with each other if the actual conditions and model premises are highly compatible and there are no measurement errors. Actually, however, differences are caused in estimated results. Now, making use of such differences, an index to evaluate the appropriateness of measurements is determined. In the [i] result of estimated airflow of the normal least squares method, [ii] result assuming the negative value of result of $i$ as airflow in reverse direction, and [iii] result of estimated airflow by a non-negative least squares method, describing the m-th estimated result of the three types of estimation methods as ${ }_{m} q_{i j}$, average value of the three types is defined with the equation (29). Variation among the three types is calculated with the variance $\sigma_{q i j}^{2}$ of the equation (30) based on the average value among the estimated results of the three types for individual airflow.

$$
\bar{q}_{i, j}=\frac{1}{3} \sum_{m=1}^{3}\left\|_{m} q_{i, j}\right\| \text { (29) } \quad \sigma_{q i j}{ }^{2}=\frac{1}{3} \sum_{m=1}^{3}\left(\left\|{ }_{m} q_{i, j}\right\|-\bar{q}_{i, j}\right)^{2}
$$


Average value $\sigma_{q 2}$ of all the airflows is obtained for this variance of airflow. Average value of all the airflows is calculated with the following equation for the solution of the non-negative least squares method.

$$
\bar{\sigma}_{q}{ }^{2}=\frac{1}{n q} \sum_{i=1}^{n+1} \sum_{j=1}^{n+1} \sigma_{q i j}{ }^{2} \text { (31) } \bar{q}=\frac{1}{n q} \sum_{i=1}^{n+1} \sum_{j=1}^{n+1}\left\|{ }_{n n} q_{i, j}\right\|
$$

If the negative flow irrationality index $R a$ of the estimated parameter of the following equation is larger than 0.5 , experimental conditions should be considered to be remarkably different from the premises of the model and improved and measurement shall be repeated.

$$
R_{a}=\frac{\bar{\sigma}_{q}}{\bar{q}}
$$

\section{CALCULATION EXAMPLES}

In order to develop a calculation program, PFTSID (tentative name), based on the above-described theory and to conduct verification of accounts, a gas concentration estimation/calculation model was prepared assuming the airflow in the multiple zone model, gas concentration was calculated and solved, and simulated measurement values were generated adding the random number of errors with normal distribution to the gas concentration predicted as described above and gas generation rate of the premise. Then, the airflow rates were estimated and errors were evaluated with these simulated measurement values.

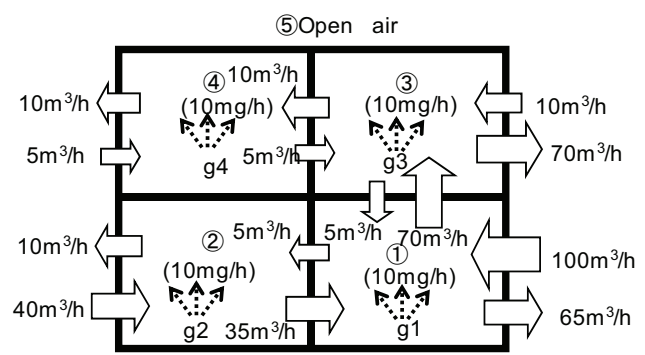

Figure1 4-zone calculation experimentation model
Table 1 True values of gas

\begin{tabular}{|c|c|c|c|}
\hline \multicolumn{4}{|c|}{ Gas dissipation rate $(\mathrm{mg} / \mathrm{h})$} \\
\hline $\begin{array}{c}1,1 g_{1} \\
10\end{array}$ & $\begin{array}{c}1,2 g_{2} \\
10\end{array}$ & $\begin{array}{c}1,3 g_{3} \\
10\end{array}$ & $\begin{array}{c}1,4 \mathrm{~g}_{4} \\
10\end{array}$ \\
\hline \multicolumn{4}{|c|}{ Gas concentration $\left(\mathrm{mg} / \mathrm{m}^{3}\right)$} \\
\hline $\begin{array}{c}1.1 .1 \mathrm{C}_{1} \\
0.07588\end{array}$ & $\begin{array}{c}1,2,1 \mathrm{C}_{1} \\
0.059051\end{array}$ & $\begin{array}{c}1,3.1 \mathrm{C}_{1} \\
0.00465\end{array}$ & $\begin{array}{c}1,4,1 \mathrm{C}_{1} \\
0.00155\end{array}$ \\
\hline $\begin{array}{c}1,1,1 \mathrm{C}_{2} \\
0.008445\end{array}$ & $\begin{array}{c}1,2,1 \mathrm{C}_{2} \\
0.22915\end{array}$ & $\begin{array}{c}1,3,1 \mathrm{C}_{2} \\
0.000517\end{array}$ & $\begin{array}{c}1,4,1 \mathrm{C}_{2} \\
0.000172\end{array}$ \\
\hline $\begin{array}{c}1,1,1 \mathrm{C}_{3} \\
0.06503\end{array}$ & $\begin{array}{c}1,2,1 \mathrm{C}_{3} \\
0.050608\end{array}$ & $\begin{array}{c}1,3,1 \mathrm{C}_{3} \\
0.126542\end{array}$ & $\begin{array}{c}1,4,1 \mathrm{C}_{3} \\
0.042181\end{array}$ \\
\hline $\begin{array}{c}1,1.1 \mathrm{C}_{4} \\
0.043353\end{array}$ & $\begin{array}{c}1,2,1 \mathrm{C}_{4} \\
0.033739\end{array}$ & $\begin{array}{c}1,3.1 \mathrm{C}_{4} \\
0.084361\end{array}$ & $\begin{array}{c}1.4 .1 \mathrm{C}_{4} \\
0.694787\end{array}$ \\
\hline
\end{tabular}
dissipation rate and gas concentration

As shown in Fig. 1, a multi-zone gas flow model was schemed assuming the airflow distribution in 4 zones. In each zone, 4 types of tracer gases would be dissipated at a rate of $10 \mathrm{mg}$ per hour. Gas concentration was calculated using a computer simulation program, NETS, assuming a steady state regardless of the zone volume, to be $100 \mathrm{~m}^{3}, 25 \mathrm{~m}^{3}, 62.5 \mathrm{~m}^{3}$, and $62.5 \mathrm{~m}^{3}$ respectively for the zone 1 through zone 4. Air tightness between the zone 2 and zone 4 is high and it was assumed that no airflow exists. In this case the number of term is one and $p=1$.

By adding errors generated based on the random number generation to the true values as shown in Table 1, assuming the standard deviation of measurement error of gas generation rate to be 1 and that of the gas concentration to be 0.001 , simulated measurement values were generated. Gas concentration was measured at 4 points in each zone. Symbol $g$ represents gas generation rate and $c$ the gas concentration, and the left and right subscript numbers are defined in the same way as described above. The right subscript numbers represent the zone numbers. The left subscript numbers represent the term No., gas type No., and position No. in the zone from the left including commas.

The conventional deterministic method obtains 20 airflows including ones which do not exist. The least squares method makes calculations for 16 and 14 airflow models depending on the existence of 
airflow between the zone 2 and zone 4 . The airflows were estimated by 3 methods; a method using negative airflows as they are according to the normal least squares method, a method assuming negative airflows to be in reverse direction, and the non-negative least squares method. Comparison of these results with true values is shown in Table 2.

Table 2 Results of various estimation methods

\begin{tabular}{|c|c|c|c|c|c|c|}
\hline $\begin{array}{l}\text { Leeward } \\
\text { i zone }\end{array}$ & $\begin{array}{c}\text { Windward } \\
\text { j zone }\end{array}$ & $\begin{array}{c}\text { Conventional } \\
\text { method }\end{array}$ & $\begin{array}{c}\text { Non-negative } \\
\text { LS, q } 20 \\
\text { assumed }\end{array}$ & $\begin{array}{c}\text { Non-negative } \\
\text { LS, q } 16 \\
\text { assumed }\end{array}$ & $\begin{array}{c}\text { Non-negative } \\
\text { LS, q } 14 \\
\text { assumed }\end{array}$ & Real q value \\
\hline 5 & 1 & 62.50 & 62.80 & 63.07 & $\begin{array}{r}63.01 \\
\end{array}$ & 65 \\
\hline 5 & 2 & 14.97 & 14.78 & 15.03 & 15.03 & 10 \\
\hline 5 & 3 & 64.57 & 64.89 & 64.14 & 64.13 & 70 \\
\hline 5 & 4 & 10.56 & 10.52 & 10.56 & 10.63 & 10 \\
\hline 1 & 5 & 94.59 & 94.59 & 94.58 & 94.58 & 100 \\
\hline 2 & 5 & 42.99 & 43.10 & 42.95 & 42.97 & 40 \\
\hline 3 & 5 & 9.70 & 10.18 & 10.13 & 10.13 & 10 \\
\hline 4 & 5 & 5.32 & 5.12 & 5.14 & 5.13 & 5 \\
\hline 1 & 2 & 33.30 & 33.28 & 33.29 & 33.28 & 35 \\
\hline 2 & 1 & 5.36 & 5.08 & 5.29 & 5.35 & 5 \\
\hline 3 & 4 & 4.74 & 4.73 & 4.72 & 4.72 & 5 \\
\hline 4 & 3 & 10.23 & 10.02 & 10.21 & 10.21 & 10 \\
\hline 1 & 3 & 5.06 & 4.66 & 5.02 & 5.03 & 5 \\
\hline 3 & 1 & 65.35 & 64.47 & 64.53 & 64.53 & 70 \\
\hline 2 & 4 & 0.08 & 0.07 & 0.07 & - & 0 \\
\hline 4 & 2 & 0.08 & 0 & 0 & - & 0 \\
\hline 4 & 1 & -0.29 & 0.19 & - & - & 0 \\
\hline 1 & 4 & -0.04 & 0 & - & - & 0 \\
\hline 3 & 2 & -0.04 & 0.19 & - & - & 0 \\
\hline 2 & 3 & -0.14 & 0 & - & - & 0 \\
\hline
\end{tabular}

Various indices for evaluating errors are shown. Table 3 shows calculation of discrepancy ratio $\beta$ to premises of models on the results of the non-negative least squares method of 14 airflows. Table 4 shows the average of the irrationality index of estimated negative airflows and $\beta$. Coefficient of determinant was also calculated, which was a value close to 1 in any model.

Table 3 Discrepancy ratio of model premises

\begin{tabular}{|c|c|r|r|r|r|r|r|}
\hline $\begin{array}{c}\text { Leeward } \\
\text { i zone }\end{array}$ & $\begin{array}{c}\text { Windward } \\
\text { jzone }\end{array}$ & $\begin{array}{c}\text { Estimated } \\
\text { q value }\end{array}$ & $\begin{array}{c}\text { Real q } \\
\text { value }\end{array}$ & $\delta$ error & $\sigma_{\lambda}$ & ${ }_{\mathrm{m}} \sigma_{\lambda}$ & $\beta$ \\
\hline 5 & 1 & 63.01 & 65 & -2.00 & 0.52 & 12.07 & 0.04 \\
\hline 5 & 2 & 15.03 & 10 & 5.03 & 0.21 & 4.06 & 0.05 \\
\hline 5 & 3 & 64.13 & 70 & -5.85 & 0.29 & 6.78 & 0.04 \\
\hline 5 & 4 & 10.63 & 10 & 0.63 & 0.05 & 1.11 & 0.05 \\
\hline 1 & 5 & 94.58 & 100 & -5.50 & 0.49 & 5.43 & 0.09 \\
\hline 2 & 5 & 42.97 & 40 & 3.01 & 0.23 & 5.36 & 0.04 \\
\hline 3 & 5 & 10.13 & 10 & 0.12 & 0.36 & 5.19 & 0.07 \\
\hline 4 & 5 & 5.13 & 5 & 0.13 & 0.03 & 3.25 & 0.01 \\
\hline 1 & 2 & 33.28 & 35 & -1.71 & 0.26 & 2.88 & 0.09 \\
\hline 2 & 1 & 5.35 & 5 & 0.34 & 0.29 & 6.71 & 0.04 \\
\hline 3 & 4 & 4.72 & 5 & -0.29 & 0.06 & 0.80 & 0.07 \\
\hline 4 & 3 & 10.21 & 10 & 0.20 & 0.04 & 3.49 & 0.01 \\
\hline 1 & 3 & 5.03 & 5 & 0.02 & 0.35 & 3.93 & 0.09 \\
\hline 3 & 1 & 64.53 & 70 & -5.46 & 0.45 & 6.40 & 0.07 \\
\hline \multicolumn{2}{|c|}{ Average value } & - & - & - & 0.26 & 4.82 & 0.06 \\
\hline
\end{tabular}

Table 4 Irrationality index of estimated negative airflow and averaqe of $\beta$

\begin{tabular}{|c|c|c|}
\hline \multicolumn{3}{|c|}{ estimated-q 16 } \\
\hline $\bar{\sigma}_{q}{ }^{2}$ & $\bar{\sigma}_{q}$ & $\bar{\beta}$ \\
\hline 0.00111 & 0.0332 & \multirow{2}{*}{0.05} \\
\cline { 1 - 2 } $\bar{q}$ & $R_{a}=\bar{\sigma}_{q} / \bar{q}$ & \multirow{2}{*}{0.00124} \\
\hline 26.794 & 0.00124 & \\
\cline { 1 - 2 } & \multicolumn{3}{|c|}{ estimated-q 14 } \\
\hline $\bar{\sigma}_{q}{ }^{2}$ & $\bar{\sigma}_{q}$ & $\bar{\beta}$ \\
\cline { 1 - 2 } 0.0000848 & 0.00921 & \multirow{2}{*}{0.06} \\
\cline { 1 - 2 } $\bar{q}$ & $R_{a}=\bar{\sigma}_{q} / q$ & \\
\hline 30.623 & 0.000301 & \\
\hline
\end{tabular}

\section{COMMENTS AND TASKS IN FUTURE}

In Table 2, there are 4 negative airflows by the conventional method, showing a relatively large difference from the non-negative least squares method. Though the estimated accuracy depending on the number of the estimated airflows by the non-negative least squares method is a little inferior in case of 20 airflow estimation to that of other methods, difference between 16 and 14 airflows is relatively small. In case of 16 airflows, one airflow is $0.07 \mathrm{~m}^{3} / \mathrm{h}$, which should be 0 . In the discrepancy ratio of model premises in Table 3, this instance is influenced only by measurement errors, premises of models are satisfactorily met, and average of $\beta$ is as small as 0.06 . The irrationality index of negative airflow $R a$ as shown in Table 4 is as large as 4 times that in case of 14 airflows having no negative airflows, in the case of 16 airflows including negative airflows. It was also known that error evaluation cannot be attained only by the coefficient of determinant. These error evaluation indices 
can be used for relative comparison but many instances should be studied in the future to determine a judgment value in advance.

In this instance, multiple terms, gas types more than the total zone number, uneven gas concentration in a zone, and influence of changes of ventilation with time, which are features of this theory and assumed to be accountable, have not yet been studied. Lack of uniformity of concentration at 4 points in a same zone of this instance is due to variation of normal probability distribution of measurement errors only. However, as the basic mathematical model has omitted the term of changes with time, proper residual analysis and error evaluation might be limited. This is a reason why most of coefficient of determinant was 1. This measurement method is essentially, however, not a measuring method to follow up the changes with time. Even if the ventilation rate may be a little changed when a door or window is opened or closed, such an advantage is expectable that average airflow rate may be estimated for a long time. Therefore, error evaluation theory as described in this paper is important also from this view point.

\section{CONCLUSIONS}

As compared with the conventional deterministic method, statistical airflow estimation, and error and reliability evaluation methods were contrived based on the least squares method. In addition to the evaluation indices of statistics, new indices of the discrepancy ratio of model premises and irrationality of estimated negative airflows were defined. Instances of the calculation program of this theory, PFTSID (tentative name), was also verified. Limits caused from the basic mathematical expression model of this theory were discussed and its significance as a long-term simple measurement method was described.

\section{ACKNOWLEDGMENTS}

We want to express our gratitude to Mr. Tomohisa Masuko, Japan Industrial Testing Co., Ltd., for coding, debugging, etc. of the calculation program.

\section{REFERENCES}

1. Hiroyasu Okuyama(1990)"System Identification Theory of the Thermal Network Model and an Application for Multi-chamber Airflow Measurement",Building and Environment, Vol.25, No.4, pp.349-363

2. Hiroyasu Okuyama(1992),"Recent Progress on the Multi-Chamber Airflow Measurement System",1992 International Symposium on Room Air Convection and Ventilation Effectiveness ISRACVE, 22-24 July, Tokyo, pp351-356

3. Stymne,Hans. and Boman,C.A.(1994),"Measurement of Ventilation and air distribution using the homogeneous emission technique a validation”, Healthy Buildings,'94,Proceedings of the 3rd International Conference

4. Hiroki Higashida, Shin-ichi Tanabe, et al(2004), "Measurement of Indoor Air Quality by Passive Method Part 10 The Field Survey in a Multi-Family Residential Building by the Simple Measurement Method for Ventilation", Summaries of Technical Papers of Annual Meeting Architectural Institute of Japan, pp.991-992, Aug.

5. Kuniaki Mihara, Hiroshi Yoshino, Kazukiyo Kumagai, Miyuki Noguchi and Yukio Yanagisawa,(2006) "Evaluation Study on Ventilation Performance by Passive Method Using Four Kinds of Tracer Gases Investigation on Accuracy by Experiment and COMIS Simulation", Summaries of Technical Papers of Annual Meeting Architectural Institute of Japan (Kanto), lecture No. 41336, pp.689-690, Sept.

6. Charls L. Lawson of the US Jet Propulsion Laboratory and Richard J. Hanson of Visual Numerics, Inc. (1974) "Solving Least Squares Problems", ISBN0-89871-356-0(pbk.) 